\title{
A complete model of keyhole and melt pool dynamics to analyze instabilities and collapse during laser welding
}

\author{
Mickael Courtois \\ ArcelorMittal Global R\&D Montataire, F-60160 Montataire, France and University of Bretagne-Sud, \\ EA 4250, LIMATB, F-56100 Lorient, France
}

Muriel Carin and Philippe Le Masson

University of Bretagne-Sud, EA 4250, LIMATB, F-56100 Lorient, France

Sadok Gaied

ArcelorMittal Global R\&D Montataire, F-60160 Montataire, France

Mikhaël Balabane

Institut Galilée Université Paris 13, F-93430 Villetaneuse, France

A complete modeling of heat and fluid flow applied to laser welding regimes is proposed. This model has been developed using only a graphical user interface of a finite element commercial code and can be easily usable in industrial R\&D environments. The model takes into account the three phases of the matter: the vaporized metal, the liquid phase, and the solid base. The liquid/vapor interface is tracked using the Level-Set method. To model the energy deposition, a new approach is proposed which consists of treating laser under its wave form by solving Maxwell's equations. All these physics are coupled and solved simultaneously in Comsol Multyphysics ${ }^{\circledR}$. The simulations show keyhole oscillations and the formation of porosity. A comparison of melt pool shapes evolution calculated from the simulations and experimental macrographs shows good correlation. Finally, the results of a three-dimensional simulation of a laser welding process are presented. The well-known phenomenon of humping is clearly shown by the model.

Key words: laser welding, level set, melt pool, fluid flow modeling, porosities, keyhole

\section{INTRODUCTION}

Although many works concerning laser welding have been published over the last $20 \mathrm{yr}$, the mechanisms responsible for weld seam defects are not yet fully established and modeled (Fig. 1). These defects, such as the collapse of the melting pool or partial penetration, are present when the metal is in liquid state and when the dynamics of solidification stops the shape of the weld joint in an unwanted geometry. To predict the appearance of these defects and explain the welded zone geometry, the numerical approach can be a useful complement to experimental investigations. Therefore many models have been developed over the last $10 \mathrm{yr}$ with an increasing complexity and less and less restrictive assumptions. To better understand the mechanisms responsible for defects, these models have to take into account the fluid flow in the melt pool and predict the dynamic shape of the keyhole which is intimately coupled with heat transfer, fluid flow, and phase transformation. Such models were first established using a 2D axially symmetric approximation which requires fewer computing resources.

One of the early works was proposed by Lee et al., ${ }^{1}$ who developed a complete heat and fluid flow model to describe spot laser welding. The model takes into account the vapor phase and the displacement of melt pool surface using the volume of fluid (VOF) method. For the first time, the dynamic instabilities in laser welding are modeled and the oscillating movement of the keyhole is shown. Ki et al. ${ }^{2}$ are the first in 2002 to propose a self-consistent three-dimensional (3D) model including the main physical phenomena involved in laser-keyhole welding. This model takes into account the solid/liquid and liquid/vapor phase transformations, the fluid flow in liquid and vapor phases, and the multiple reflections of laser beam in the keyhole. The modeling of the recoil pressure has been carefully considered and a representation of the Knudsen layer is proposed. The dynamic evolution of the liquid/vapor interface is tracked selfconsistently using the Level-Set method. However, the calculations are performed on a supercomputer, therefore not readily accessible for common users. Furthermore, the influence of welding parameters such as welding speed was not investigated in this study.

More recently, with the improvement of computation capabilities, many authors have proposed similar 3D models. These models describe the evolution of the free surface of the melt pool and the defects apparition is beginning to be addressed. Geiger et $a l^{3}$ have developed a complete multiphysics model based on the VOF approach. This model has successfully reproduced the periodic keyhole oscillations. The model is used to analyze the influence of the gap in overlap welding. The role of the zinc vapor in the apparition of residual porosities is also addressed. Pang et al. ${ }^{4}$ have proposed a 


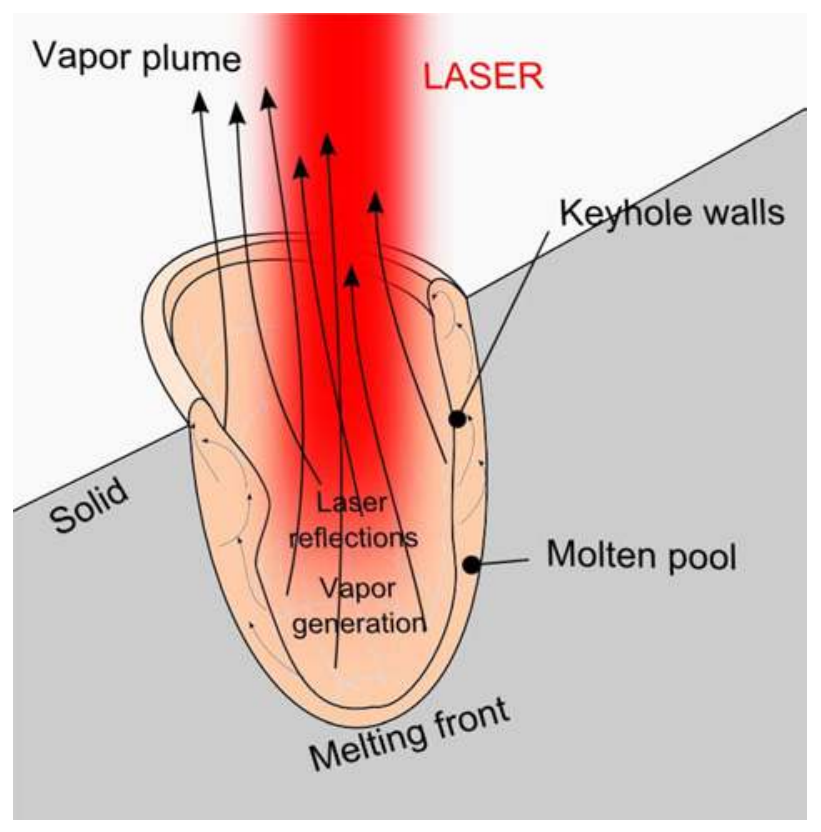

FIG. 1. Schematic diagram of phenomena in spot laser welding.

similar model to predict the keyhole formation using a LevelSet method. The mechanisms of keyhole instabilities are investigated under different heat input conditions. Cho et al. ${ }^{5}$ have used an equivalent $3 \mathrm{D}$ model to demonstrate that the Marangoni effect in laser welding configurations can be neglected. In most of these models, the effect of the recoil pressure is modeled by a force applied at the surface of the liquid but the vapor generation is not really treated. This force is generally calculated based on the model proposed by Knight. ${ }^{6}$ The velocity field in the gas is very rarely discussed and only Cho et al..$^{5}$ have proposed a simplified study to analyze the influence of the shear stress induced by the metallic vapor on the keyhole wall. Nevertheless, it has been shown experimentally that the rear front of the keyhole is strongly affected by the vapor ejection and can be at the origin of the humping phenomena with high speed of welding, ${ }^{7}$ or can be responsible of spatters ejections caused by keyhole closing. It seems that the shear stress induced by the metallic vapor cannot be neglected in all configurations and should be more discussed. Tan et al. ${ }^{8}$ have recently proposed a $3 \mathrm{D}$ model to investigate the dynamics of keyhole using a Level-Set method. They are the first to account for the plume attenuation of laser due to particle absorption/scattering in the case of $\mathrm{Nd}$ :YAG laser welding process. For that purpose, a conservation equation of chemical species is coupled with the dynamic keyhole model to predict the distribution of iron species in the keyhole plume. Note that this model is based on a sharp interface formulation in order to apply the jump conditions at the interface wall, including capillary force, Fresnel absorption of laser energy, surface radiation, evaporation, and recoil pressure.

It appears that in most of the models proposed in the literature, ${ }^{2,5}$ the multiple reflections of laser beam are taken into account using a ray-tracing model. In this method, the laser beam is discretized in a finite number of rays and each ray carries a part of the incident laser energy. Furthermore, as the methods chosen to track the dynamic change of keyhole wall are fixed mesh methods (Level-Set, VOF), specific algorithms are required such as sharp interface method.

The outline of this paper is summarized as follows. First, heat and fluid flow equations are presented for the three phases of the matter (gas, liquid, and solid). The Level-Set method used to track the interface between liquid and gas is briefly presented. A rarely used method to take into account the vapor generation is also described by modifying conservation equation. This method introduces implicitly the recoil pressure and the vapor shear stress. An original method to take into account polarization and multiple reflections is then briefly presented. Finally the model is used to simulate the dynamic formation of the keyhole and investigate the mechanisms responsible for the collapse of the melt pool in a 2D axially symmetric approximation describing a static laser shot. The model is validated against experiments obtained with a Nd:YAG laser. Although this configuration is far from industrial configurations, it is an essential step before a $3 \mathrm{D}$ modeling in order to validate the numerical model. Finally, this model, implemented in the commercial code COMSOL Multiphysics ${ }^{\circledR}$, is transposed to a 3D geometry to show the ability of the model to simulate humps for the case of moving beam welding.

\section{HEAT AND FLUID FLOW MODEL}

Both the gas and liquid metal are considered Newtonian and incompressible, except for the buoyancy term in the momentum equation. Flows are considered laminar. The conservation equations of energy (1), mass (2), and momentum (3) are solved in the three phases (vapor, liquid, and solid) and can be written as follows:

$$
\begin{aligned}
& \rho c_{\mathrm{p}}\left[\frac{\partial T}{\partial t}+\vec{\nabla} \cdot(\vec{u} T)\right]= \vec{\nabla} \cdot(\lambda \vec{\nabla} T)+S_{\text {laser }}+Q_{\text {vap }}, \\
& \vec{\nabla} \cdot \vec{u}=0 \\
& \rho\left(\frac{\partial \vec{u}}{\partial t}+\vec{u} \cdot(\vec{\nabla} \cdot \vec{u})\right)= \vec{\nabla} \cdot\left[-p I+\mu\left(\vec{\nabla} \vec{u}+(\vec{\nabla} \vec{u})^{\mathrm{T}}\right)\right] \\
&+\rho \vec{g}-\rho_{l} \beta_{l}\left(T-T_{\text {melting }}\right) \vec{g} \phi \\
&+K \vec{u}+\gamma \vec{n} \kappa \delta(\phi) .
\end{aligned}
$$

With $T$ the temperature, $t$ the time, $\rho$ the density, $c_{\mathrm{p}}$ the specific heat capacity, $\lambda$ the thermal conductivity, $S_{\text {laser }}$ and $Q_{\text {vap }}$ heat sources to represent, respectively, the energy of the laser detailed in Sec. III and the energy loss by evaporation [Eq. (6)], $\vec{u}$ the velocity vector, $p$ the pressure, $I$ the identity matrix, $\mu$ the dynamic viscosity, $(.)^{\mathrm{T}}$ the transposed matrix, $K \vec{u}$ the Darcy damping force to cancel the velocity if the temperature is lower than the melting temperature $\mathrm{T}_{\text {melting, }} \vec{g}$ the gravity acceleration, $\beta_{l}$ the volume expansion coefficient of liquid, $\phi$ the Level-Set function ( $\phi=0$ in the gas, $\phi=1$ in the dense phase), $\gamma$ the surface tension coefficient, $\vec{n}$ the surface normal vector, $\kappa$ the curvature, and $\delta(\phi)$ the delta function of the Level-Set values. Buoyancy effect is neglected only in the vapor phase due to high vapor velocity. The surface tension coefficient $\gamma$ is assumed to be constant in this model which means that the Marangoni effect is neglected. This assumption is based on the work done by Cho et al. ${ }^{5}$ 
The Level-Set method is chosen to track the liquid-gas interface, since it can treat easily the complex geometric changes of the interface, and so the formation of porosity. This method uses a fixed mesh and defines a variable $\phi$ in the entire computational domain. This variable takes the value of 0 in the vapor phase and 1 in the liquid (and solid) phase. This variable is used to define the thermophysical properties of each phase and the interface is identified by locating the isovalue $\phi=0.5$. The movement of the liquid/gas interface is realized by advecting the $\phi$ variable using the velocity field calculation. The Level-Set equation is given by Eq. (4) where $\varepsilon_{\mathrm{ls}}$ defines the distance of transition and $\gamma 1 \mathrm{~s}$ the speed of re-initialization. Near the interface, the $\phi$ variable varies progressively with a smooth step function, inducing the property variation from one phase to the other. The thickness of this transition must be small enough to represent the interface accurately but not too small, in order to avoid numerical convergence problems.

The boundary conditions at the liquid-gas interface are introduced into the model as extra source terms for the governing equations using the $\phi$ variable position. So, the laser energy deposition at the surface of the metal is treated through the source term $S_{\text {laser }}$ in the heat equation deduced from electromagnetic calculation (see Sec. III). The latent heat of fusion $\mathrm{L}_{\mathrm{f}}$ is included in an apparent heat capacity by adding a Gaussian function centered at the melting temperature $T_{\text {melting }}$ and with $\left(T_{1}-T_{\mathrm{s}}\right)$ width (5), where $T_{1}$ and $T_{\mathrm{s}}$ represent the liquidus and solidus temperatures, respectively. This method is commonly used in fixed grid techniques for phase change. The energy loss due to evaporation is described by Eq. (6) with $L_{\mathrm{v}}$ the latent heat of evaporation, $\dot{m}$ the mass flux of evaporation described by Eq. (11), and $\delta(\phi)$ the delta function of the Level-Set values. The Darcy damping force in Eq. (3) introduces an isotropic permeability $\mathrm{K}$ derived from the Kozeny-Carman equation (8), and is widely used to treat the mushy region. ${ }^{2,4,5}$ This term depends on the liquid fraction $f_{1}$ and is present only if the temperature is below the melting point. The solid can then be viewed as an extremely viscous fluid. In Eq. (7), $C$ is a constant representing the mushy zone morphology and $\mathrm{b}$ a parameter to avoid the division by zero

$$
\begin{aligned}
& \frac{\partial \phi}{\partial t}+\vec{u} \cdot \nabla \phi=\gamma_{\mathrm{ls}} \nabla \cdot\left(\varepsilon_{\mathrm{ls}} \nabla \phi-\phi(1-\phi) \frac{\nabla \phi}{|\nabla \phi|}\right), \\
& c_{\mathrm{p}}^{\mathrm{eq}}=c_{\mathrm{p}-\mathrm{s}}+L_{\mathrm{f}} \frac{e^{-\left(\frac{\left(T-T_{\mathrm{meling}}\right)^{2}}{\left(T_{1}-T_{\mathrm{s}}\right)^{2}}\right)}}{\sqrt{\pi\left(T_{1}-T_{\mathrm{s}}\right)^{2}}}, \\
& Q_{\text {vap }}=-L_{\mathrm{v}} \dot{m} \delta(\phi), \\
& \left.K=-C \frac{\left(1-f_{1}\right)^{2}}{f_{1}^{3}+b}\right) \\
& f_{\mathrm{l}}=\left\{\begin{array}{l}
0 \text { for } T<T_{\mathrm{s}} \\
\frac{T-T_{1}}{T_{1}-T_{\mathrm{s}}} \text { for } T_{\mathrm{s}}<T<T_{1} \\
1 \text { for } T>T_{\mathrm{l}} .
\end{array}\right.
\end{aligned}
$$

Generally, the vaporization-induced recoil pressure is calculated using an empirical formulation. ${ }^{1,3,5}$ However, a more physical way is to introduce a source term into the continuity equation (9) which is proportional to the evaporation flow rate and nonzero only at the interface. This approach has been used by Sajid ${ }^{9}$ and Esmaeeli and Tryggvason ${ }^{10}$ in the case of water boiling using the Level-Set method and applied recently for laser welding by $\mathrm{Ki}$ et al. ${ }^{11}$ With this method, a single field formulation incorporates the effect of the interface in the equations as delta function source terms, which act only at the interface. In regard to mass conservation, incompressibility is satisfied within each phase but does not hold at the interface because of fluid expansion due to liquid-vapor phase change. An additional term is, therefore, introduced into the continuity equation (9) as described in Refs. 9-11, which can be viewed as a local interfacial mass transfer source/sink due to expansion/contraction upon phase change. If there is locally no phase change, then this equation reduces to the customary incompressible constraint. Note that this term will induce implicitly a recoil pressure in the momentum equation. The Level-Set transport equation is also modified by adding a source term (10). These source terms $(9,10)$ create evaporation phenomena and depend on the mass flow rate $\dot{m}$ (11) which is deduced from the local temperature and the saturated vapor pressure (12). ${ }^{12}$

$$
\begin{aligned}
& \vec{\nabla} \cdot \vec{u}=\dot{m} \delta(\phi)\left(\frac{\rho_{1}-\rho}{\rho^{2}}\right) \\
& \frac{\partial \phi}{\partial t}+\vec{u} \cdot \vec{\nabla} \phi-\dot{m} \delta(\phi)\left(\frac{\phi}{\rho_{1}}+\frac{1-\phi}{\rho_{\mathrm{v}}}\right) \\
& =\gamma_{\mathrm{ls}} \nabla \cdot\left(\varepsilon_{\mathrm{ls}} \nabla \phi-\phi(1-\phi) \frac{\nabla \phi}{|\nabla \phi|}\right), \\
& \dot{m}=\sqrt{\frac{m}{2 \pi k_{\mathrm{b}}}} \frac{p_{\mathrm{sat}}(T)}{\sqrt{T}}\left(1-\beta_{\mathrm{r}}\right) \\
& p_{\text {sat }}(T)=p_{\mathrm{a}} \exp \left[\frac{m L_{\mathrm{v}}}{k_{\mathrm{b}} T_{\text {vap }}}\left(1-\frac{T_{\mathrm{vap}}}{T}\right)\right] .
\end{aligned}
$$

With $\mathrm{m}$ the atomic weight of iron, $\mathrm{k}_{\mathrm{b}}$ the Boltzmann constant, $p_{\text {sat }}(T)$ the saturated vapor pressure, and $\beta_{\mathrm{r}}$ the retrodiffusion coefficient, assumed to be equal to 0 in a first approximation.

\section{LASER REFLECTIONS, A WAVE DESCRIPTION}

The multiple reflections of the laser beam inside the keyhole are generally treated using the ray-tracing method. This method calculates the laser beam path along the keyhole and the energy lost by the beam at each reflection. These calculations are performed using specific algorithm to be fully coupled with a finite element code in $2 \mathrm{D}$ and $3 \mathrm{D}$. In this paper, a new strategy is briefly presented to represent the multiple reflections of the laser beam. This approach consists in treating this problem as a wave propagation problem by solving the Maxwell's equations. The process of multiple reflections can then be implemented directly in the graphical user 
interface of COMSOL Multiphysics ${ }^{\circledR}$. More details of this original model are available in Ref. 13.

Considering the travel time of the laser beam in the keyhole $(<1 \mathrm{~ns})$ compared to the characteristic times of heat transfer and fluid flow $(>1 \mu \mathrm{s})$, the approximation of a stationary wave is fully justified and the electromagnetic problem can be solved with a stationary solver for each time step of the heat and fluid flow problem (Fig. 2).

The Maxwell's equations can be written in their general form (no microwave or induction simplifications) for a stationary electrical field, as follows:

$$
\begin{aligned}
& \frac{\nabla \vec{E}}{\mu_{\mathrm{r}}}+k_{0}^{2}\left(\varepsilon_{\mathrm{r}}-\frac{j \sigma}{\omega}\right) \vec{E}=0, \\
& k_{0}=\frac{\omega}{c_{0}}=\omega \sqrt{\varepsilon_{0} \mu_{0}} .
\end{aligned}
$$

With $\vec{E}$ the electrical field, $\mu_{\mathrm{r}}$ the relative permeability, $\varepsilon_{\mathrm{r}}$ the relative permittivity, $\sigma$ the electrical conductivity, $\omega$ the angular frequency, $k_{0}$ the wave number of free space, and $c_{0}$ the speed of wave in free space. This equation is solved in the entire calculation domain, for gas, liquid, and solid phases. Depending on the electromagnetic properties of the medium, the wave will propagate easily in the gas, which represents a totally transparent material, but will be partially reflected when encountering the dense material, such as steel. Here, the liquid and solid phases are assumed to have the same properties. The change of electromagnetic properties at the interface between the gas and dense phase is realized with the Level-Set value which is already used for the heat and fluid flow problem.

An electric field with a top-hat distribution is set at the top of the domain (15) to simulate the laser beam. A scattering boundary condition is chosen in the Comsol Multiphysics $^{\circledR}$ software. With this condition, an incoming electric field is applied at the top boundary, which is also transparent for a potential outgoing wave

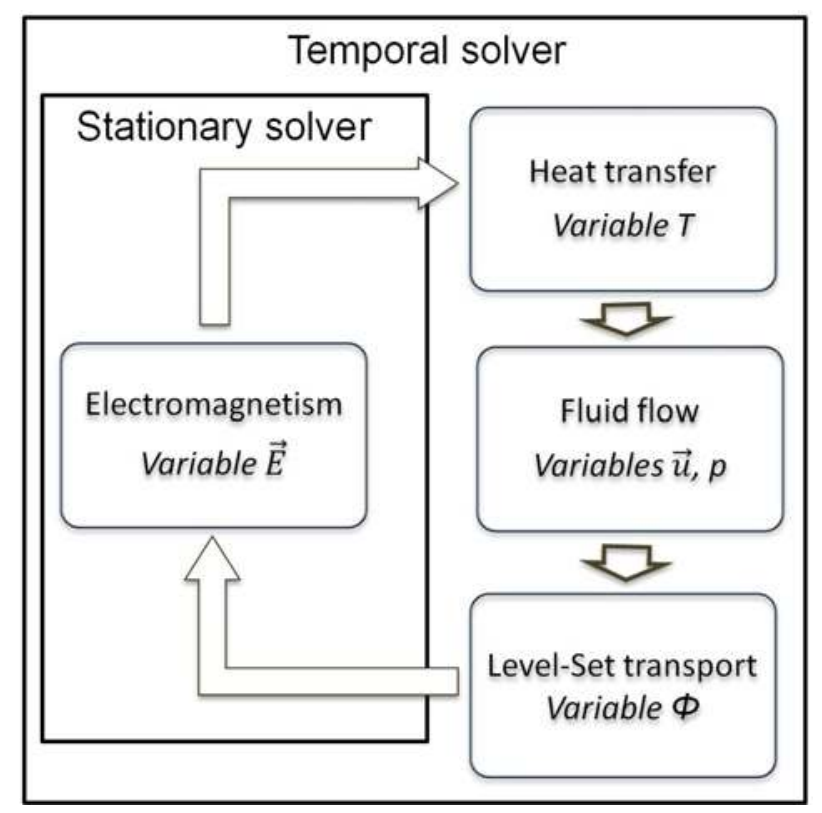

FIG. 2. Schematic diagram of the resolution process.

$$
\begin{aligned}
& \left.E_{z}(x, y)=E_{0} \sqrt{\frac{w_{0}}{w(x)}} H(y) \cos \quad \omega_{0}-k x+\eta(x)-\frac{k y^{2}}{2 R(x)}\right) \\
& w(x)=w_{0} \sqrt{1+\left(\frac{x}{x_{0}}\right)^{2}} \\
& \eta(x)=\frac{1}{2} \arctan \left(\frac{x}{x_{0}}\right) \\
& \left.R(x)=x \quad 1+\left(\frac{x_{0}}{x}\right)^{2}\right)
\end{aligned}
$$

where $x$-axis is the direction of propagation, $\mathrm{E}_{0}$ is the electric field amplitude, $H(\mathrm{y})$ an Heaviside function to define the top-hat distribution equal to 1 if $\left(y<w_{0}\right)$ and to 0 if $\left(y>w_{0}\right)$ with a smooth transition of $100 \mu \mathrm{m}, \mathrm{w}_{0}$ is the minimum waste of the beam, $w(x)$ the width of beam, and $\eta(x)$ the Gouy phase. The wave front is spherical with a $R(x)$ radius but becomes plane near the focal point $\mathrm{x}_{0}$. In Eq. (15), the distribution is Gaussian in $y$ direction with the exponential term. This term can be changed to describe another distribution like a top-hat distribution. Note that the keyhole wall is inside the calculation domain and the reflections are induced by the change of electromagnetic properties at the interface between the dense phase and gas.

The choice of the electromagnetic properties controls the absorption coefficient. Depending on this choice, the medium can vary from a totally transparent material like air to a totally reflective material. In the case of Nd:Yag laser interaction with steel, the absorption-reflection is partial at the interface gas/steel. The Fresnel laws are widely used ${ }^{1-5}$ to predict the absorption coefficient on metals. To predict accurately the propagation of the Nd:YAG laser beam using the Maxwell's equations, the mesh size must be smaller than the wavelength of the laser $(1.06 \mu \mathrm{m})$, thus ideally a mesh size of $0.2 \mu \mathrm{m}$. Furthermore, to describe the absorption of the wave at the interface gas/metal, the size of the elements must also be smaller than the skin depth. Typically, for steel and $\mathrm{Nd}$ :YAG laser, the skin depth is only a few nanometers. Such fine mesh is too expensive in regard to the dimension of the keyhole, which is of order of millimeter. To overcome this difficulty and obtain acceptable calculation times, a wavelength of $50 \mu \mathrm{m}$ is taken instead of $1.06 \mu \mathrm{m}$ in Nd:YAG and the physical phenomena in skin depth are not represented. With this choice, the same mesh can be used for the electromagnetic problem and the heat and fluid flow problem, the coupling between these equations are then facilitated. The use of a $50 \mu \mathrm{m}$ wavelength instead of a $1.06 \mu \mathrm{m}$ wavelength implies to modify the properties of the dense phase to obtain the same absorption coefficient as a function of the angle of incidence than expected. The values of the modified parameters are identified using a simple model predicting the input and output of waves after reflection on the dense phase. The electromagnetic model used here therefore verifies the Fresnel laws (Fig. 3).

The resolution of Maxwell's equations gives the electric field and the magnetic field at each point of the computational 


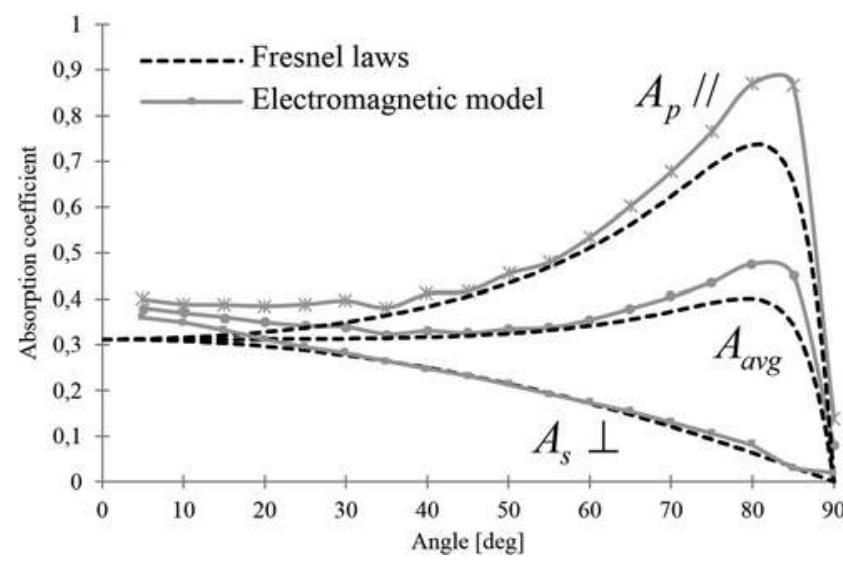

FIG. 3. Comparison between Fresnel laws (steel; $1.06 \mu \mathrm{m}$ ) and electromagnetic model $(50 \mu \mathrm{m})$.

domain. The Poynting vector can be calculated in order to obtain the distribution of the energy deposited at the keyhole wall. It is also possible to use the electromagnetic energy density, which is given by

$$
\Gamma=0.5 \vec{D} \cdot \vec{E}+0.5 \vec{B} \cdot \vec{H}
$$

With $\vec{D}$ the electric displacement field, $\vec{B}$ the magnetic induction, and $\vec{H}$ the magnetic field. This electromagnetic energy density is used to calculate the source term $S_{\text {laser }}$ in the energy equation (20). As explained before, the wavelength of the incoming electric field has been modified to overcome numerical constraints.

Therefore, a normalization of the electromagnetic energy density is needed to keep the correct cartography of the laser deposition [ratio in Eq. (20)]. This ratio is equal to zero far from the interface

$$
\begin{aligned}
& S_{\text {laser }}=\frac{\delta(\phi) \Gamma}{\int_{v}(\delta(\phi) \Gamma) d v} P_{\text {laser }} \eta \\
& \eta=1-\left(\eta_{\mathrm{o}}+\eta_{\mathrm{b}}+\eta_{\mathrm{g}}\right)
\end{aligned}
$$

$$
\begin{aligned}
& \eta_{\mathrm{b}}=\int_{\text {boud }} \frac{-\left(\Gamma_{\text {output }}-\Gamma_{\text {input }}\right)}{\Gamma_{\text {input }}} \\
& \eta_{\mathrm{g}}=\frac{0.15}{1+e^{(-1000 t+5)}}
\end{aligned}
$$

$P_{\text {laser }}$ represents the power of the laser beam and $\eta$ is the laser efficiency (21). This parameter includes three mains losses: The optical loss from the machine $\eta_{o}$ is estimated at $5 \%$. The boundary losses $\eta_{\mathrm{b}}(22)$ are due to the reflected waves which escape the computational domain. Finally, the gas losses $\eta_{g}$ represent the vapor plume absorption. Modeling several centimeters above the keyhole will be too expensive. Experimental and numerical investigations evaluate these losses generally between $10 \%$ and $20 \%^{7,14,15}$ in the case of steel welding with a $1.06 \mu \mathrm{m}$ laser. Based on these works, an increasing sigmoidal function is chosen with a maximum value of $15 \%$ (23). Temporal variations after the plume establishment are then not taken into account.

\section{RESULTS AND DISCUSSION}

The model is used to simulate a static laser shot of $25 \mathrm{~ms}$ followed by cooling. A Nd:YAG laser with a focal spot diameter of $600 \mu \mathrm{m}$ at the focal position is used for calculations. The sample is a disk of $2 \mathrm{~mm}$ in radius and $1.8 \mathrm{~mm}$ in thickness in DP 600 steel. The thermophysical properties are listed in Table I. The properties in the solid phase are taken from ArcelorMittal data and are similar to properties given in Ref. 16. Note that the value of the surface tension coefficient was under-estimated in this first study for numerical convenience. Using a more realistic value leads to strong forces acting at the singularity solid-liquid-vapor point and too great an effort on the solid part. The computations are performed for three laser power (1000, 1250, and $1500 \mathrm{~W})$ and with a top-hat distribution for the beam. The transient equations of heat transfer, mass, momentum and Level-Set are solved simultaneously in a 2D axial symmetric coordi-

\begin{tabular}{|c|c|c|}
\hline Property (unit) & Symbol & Value or reference \\
\hline Thermal conductivity of solid/liquid/gas $\left(\mathrm{W} \cdot \mathrm{m}^{-1} \cdot \mathrm{K}^{-1}\right)$ & $k_{\mathrm{s} / 1 / \mathrm{g}}$ & $\mathrm{f}(T)^{16} / 3.75 .10^{-3} T+23.25 / 0.12$ \\
\hline Density of solid/liquid/gas $\left(\mathrm{kg} \cdot \mathrm{m}^{-3}\right)$ & $\rho_{\mathrm{s} / 1 / \mathrm{g}}$ & $\mathrm{f}(T)^{16} / 7287 / 1$ \\
\hline Specific heat of solid/liquid/gas $\left(\mathrm{J} \cdot \mathrm{kg}^{-1} \cdot \mathrm{K}\right)$ & $C \mathrm{p}_{\mathrm{s} / 1 / \mathrm{g}}$ & $\mathrm{f}(T)^{16} / 573 / 373$ \\
\hline Dynamic viscosity of liquid/gas $(\mathrm{Pa} \cdot \mathrm{s})$ & $\mu_{1 / \mathrm{g}}$ & $5 \times 10^{-3} / 1 \times 10^{-5}$ \\
\hline Latent heat of fusion $\left(\mathrm{J} \cdot \mathrm{kg}^{-1}\right)$ & $L_{\mathrm{f}}$ & $2.54 \times 10^{5}$ \\
\hline Latent heat of vaporization $\left(\mathrm{J} \cdot \mathrm{kg}^{-1}\right)$ & $L_{\mathrm{v}}$ & $6.1 \times 10^{6}$ \\
\hline Liquidus temperature $(\mathrm{K})$ & $T_{1}$ & 1808 \\
\hline Solidus temperature $(\mathrm{K})$ & $T_{\mathrm{s}}$ & 1788 \\
\hline Melting temperature $(\mathrm{K})$ & $T_{\text {melting }}$ & $\left(T_{1}+T_{\mathrm{s}}\right) / 2$ \\
\hline Vaporization temperature $(\mathrm{K})$ & $T_{\text {vap }}$ & 3134 \\
\hline Surface tension coefficient $\left(\mathrm{N} \cdot \mathrm{m}^{-1}\right)$ & $\gamma$ & 0.15 \\
\hline Coefficients in Darcy's law (8) & $C / \mathrm{b}$ & $1.10^{6} / 1.10^{-3}$ \\
\hline Relative permeability of liquid/gas & $\mu_{\mathrm{r}}$ & $1 / 1$ \\
\hline Identified electric conductivity of liquid/gas (S/m) & $\sigma$ & $0 / 0$ \\
\hline Identified complex relative permittivity_liquid/gas & $\varepsilon_{\mathrm{r}}$ & $86(1-i \tan (0.6)) / 1$ \\
\hline
\end{tabular}
nates with the stationary Maxwell's equations.

The entire model is developed with Comsol Multiphysics ${ }^{\circledR}$ software using the computational fluid dynamics module to

TABLE I. Thermophysical properties of the three phases. 
solve Level-Set transport equation and radio frequency module to solve Maxwell's equations. The model has 30000 quadratic triangular elements of $10 \mu \mathrm{m}$ for electromagnetic equation and 20000 linear triangular elements of $20 \mu \mathrm{m}$ for the other equations (heat, fluid flow, Level-Set). The resolution on 6 cores at $3.46 \mathrm{GHz}$ requires $10 \mathrm{~GB}$ ram and around $16 \mathrm{~h}$ to describe $10 \mathrm{~ms}$ of process.

Figure 4 shows the creation of the keyhole at different times for a $1500 \mathrm{~W}$ laser applied for $20 \mathrm{~ms}$ with an efficiency $\eta$ calculated by Eq. (21). The absorption coefficient is gradually increasing from $0 \%$ to $55 \%$ up to $10 \mathrm{~ms}$ and then stays constant. A strong mass flow rate of vapor escapes from the keyhole with velocities of around $50 \mathrm{~m} \cdot \mathrm{s}^{-1}$ and interacts with the melt pool. A main vortex is observed in the upper part of the melt pool (Fig. 4). Velocities are approximately equal to $0.5 \mathrm{~m} / \mathrm{s}$ at the interaction interface and smaller near the fusion front $(<0.1 \mathrm{~m} / \mathrm{s})$. So, maximum velocities are found at the surface of the liquid. Note that the results are the same when buoyancy effect is neglected in the liquid phase. This indicates that the only additional driving force acting on the fluid flow in the liquid metal (in addition of the recoil pressure) comes from the shear stress from the vapor plume on the melt pool. Without shear stress, the maximum velocity is in the melt pool rather than at the surface. The effect is relatively weak in this configuration but, it can play a major role in 3D configuration with an inclined keyhole. Thermal gradients are very elevated near the keyhole surface
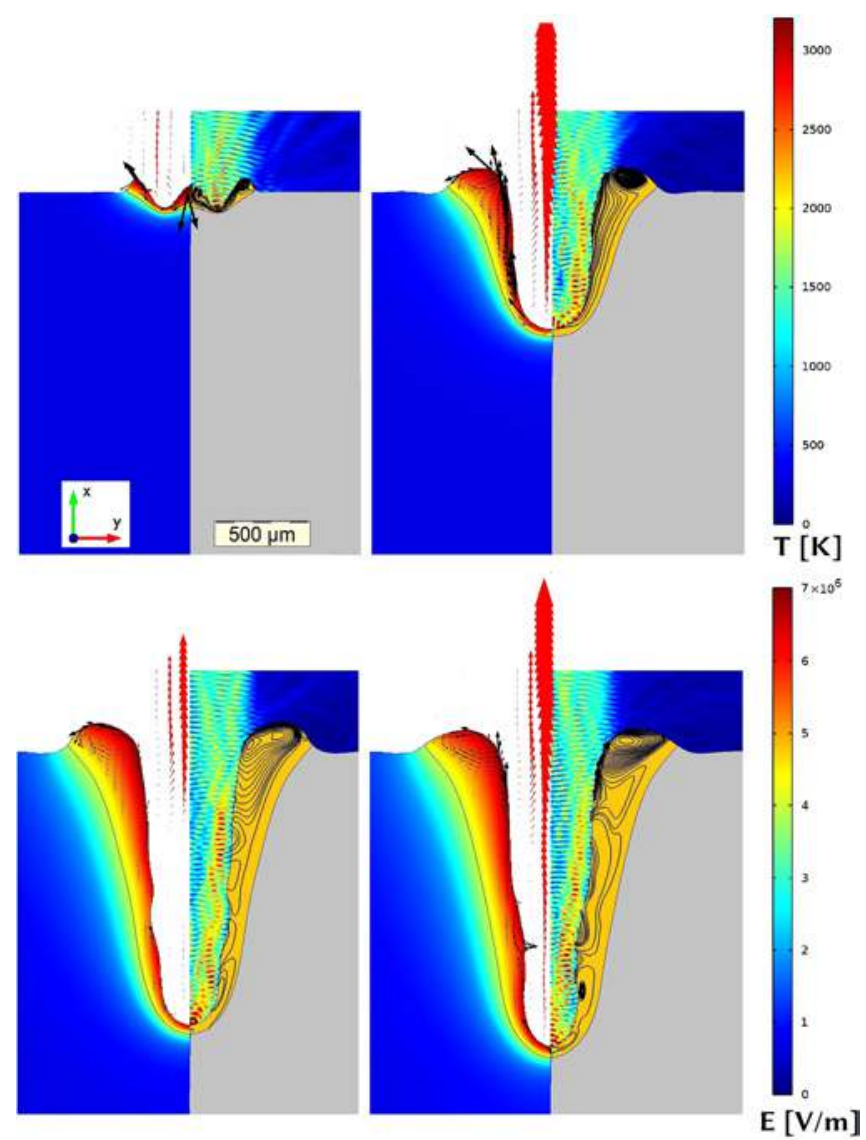

FIG. 4. Melt pool shapes, temperature field (left), velocity vector fields (left), and laser electric field (right). $P_{\text {laser }}=1500 \mathrm{~W}$ (stopped at $20 \mathrm{~ms}$ )-Interactions times: $1,7,17$, and $20 \mathrm{~ms}$. and the thickness of liquid is very narrow at the beginning. During all the irradiation process, the minimum thickness of liquid varies from 40 to $80 \mu \mathrm{m}$. After $7 \mathrm{~ms}$, the size of the melt pool becomes larger and a vortex appears at the top of the melt pool due to the interaction with the vapor phase. Up to $10 \mathrm{~ms}$, the keyhole is relatively stable and the surface is smooth. Then, the keyhole becomes deep enough and the recoil pressure cannot compensate the surface tension along the entire surface. Oscillations are observed leading to a potential beginning of collapse of the keyhole. At high power and long times of irradiation, the keyhole is not stable anymore. Under the effect of recoil pressure, the melt pool is ejected upward, and then it collapses on itself. As long as irradiation is applied, the liquid is immediately removed upward leading to repeated oscillations. These successions of collapse and repulsion can be extremely fast, and induce the creation of a corrugated surface. This phenomenon is periodic and the keyhole wall has many undulations.

At high laser power, the model presents a complex mechanism of melt pool collapse during cooling. When the laser is shut off, vaporization and therefore recoil pressure are immediately stopped. Gravity and surface tension are the only forces acting and lead to a fast collapse of the keyhole. The time of closure of the keyhole is lower than $3 \mathrm{~ms}$. If the keyhole is sufficiently deep, most of the liquid is maintained in the upper part by recoil pressure during irradiation. The small quantity of liquid in the lower part is less mobile. In general, the upper part collapses from above the keyhole and the lower part moves too slowly to fill the keyhole from the bottom. At these laser powers, a gas cavity is almost always formed during collapse. The presence of a residual porosity at the end of the solidification is a function of the elevation of formation of the bubble and its size for a given solidification time. In Fig. 5, the laser is stopped and a bubble appears

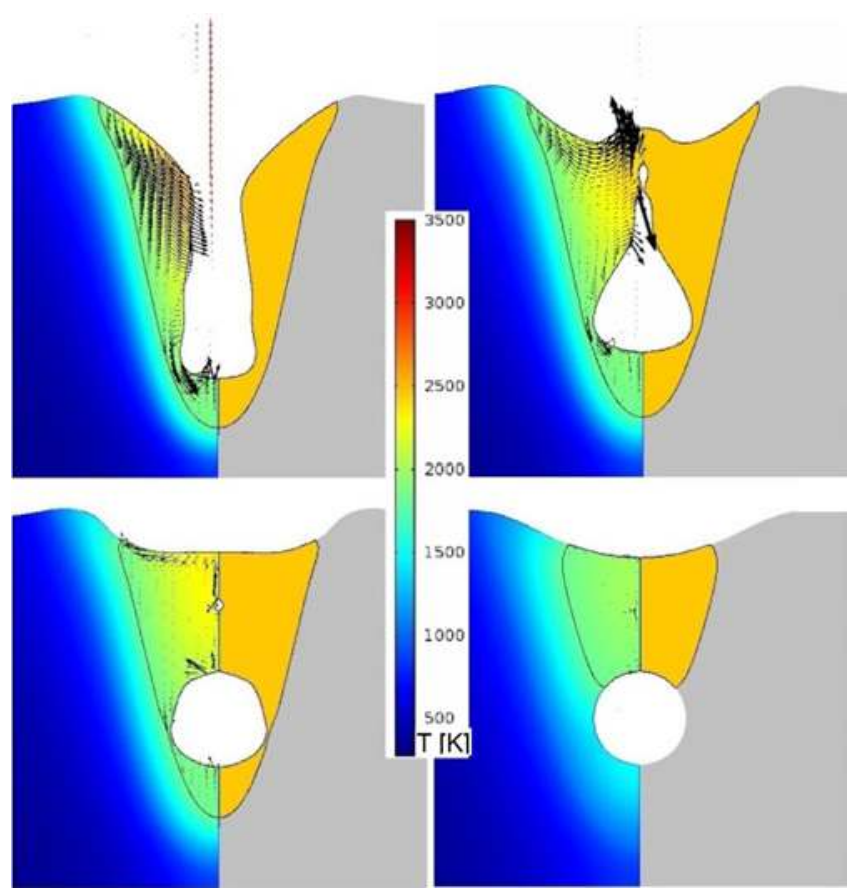

FIG. 5. Melt pool shapes and temperature field during the keyhole collapse and porosity formation at $21.2,21.7,22.5$, and $26 \mathrm{~ms}$; $\left(P_{\text {laser }}=1500 \mathrm{~W}\right)$. 


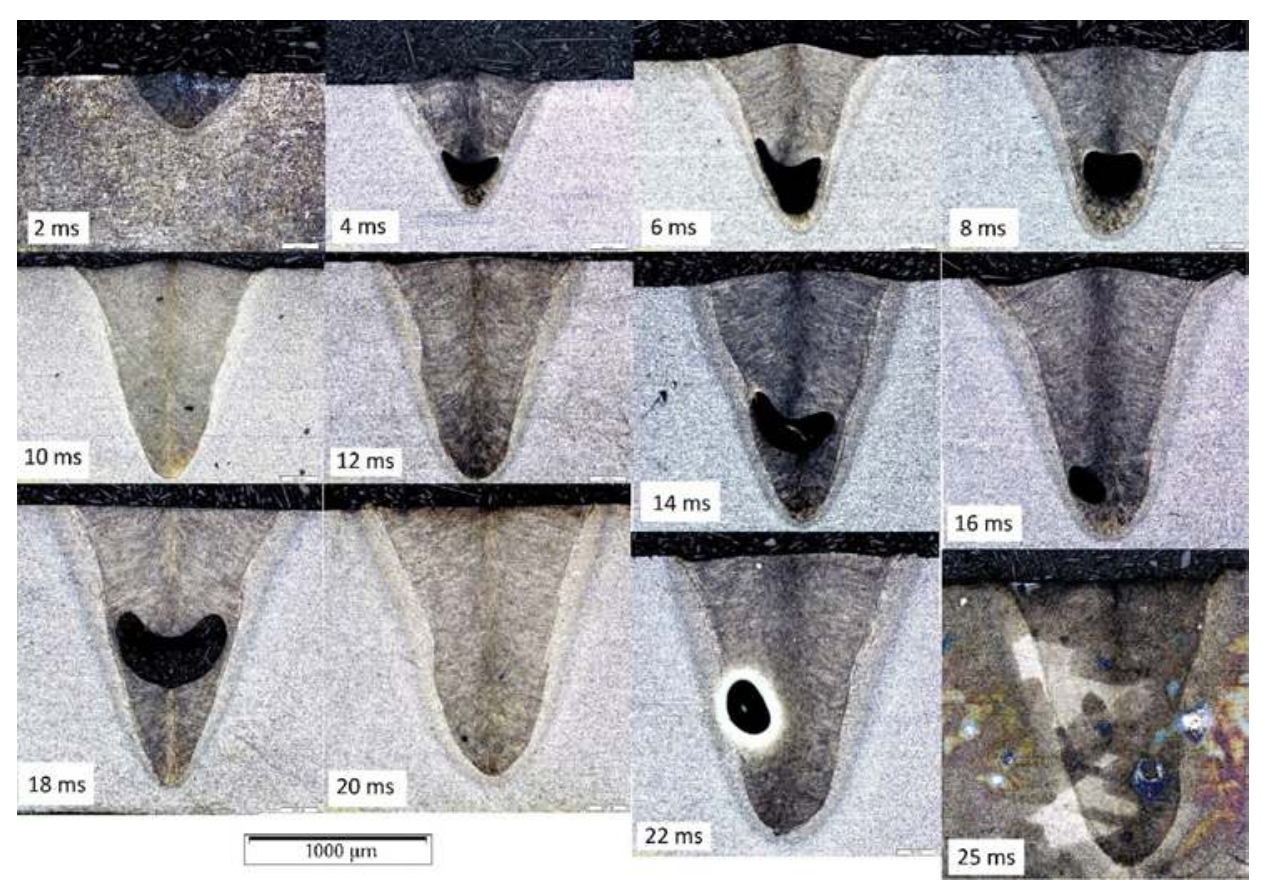

FIG. 6. Macrographs of fusion zones; variable interaction time; $P_{\text {laser }}=1500 \mathrm{~W}$.

at the bottom of the melt pool. In that case, it does not have the time to reach the melt pool surface before the full solidification and porosity is then formed. In other cases the bubble is formed in the upper part and can rises to the surface by Archimedes' principle. The fusion zone appears free from defects. The appearance of a residual porosity is very variable and is linked to the shape of the capillary at the laser stop time. Indeed, walls of the capillary are instable and oscillating. Modify a little the stop time can lead to a completely different mechanism of collapse. This small difference can create the bubble more or less high and can favor the appearance of a residual porosity. This "probability" of a porosity is also found experimentally as shown in Sec. V.

\section{EXPERIMENTAL STUDY}

A series of experiments was performed at the PIMM laboratory to achieve two goals. The first one is to obtain a transient evolution of welded zone geometry to validate the numerical model. The second is to identify the operating

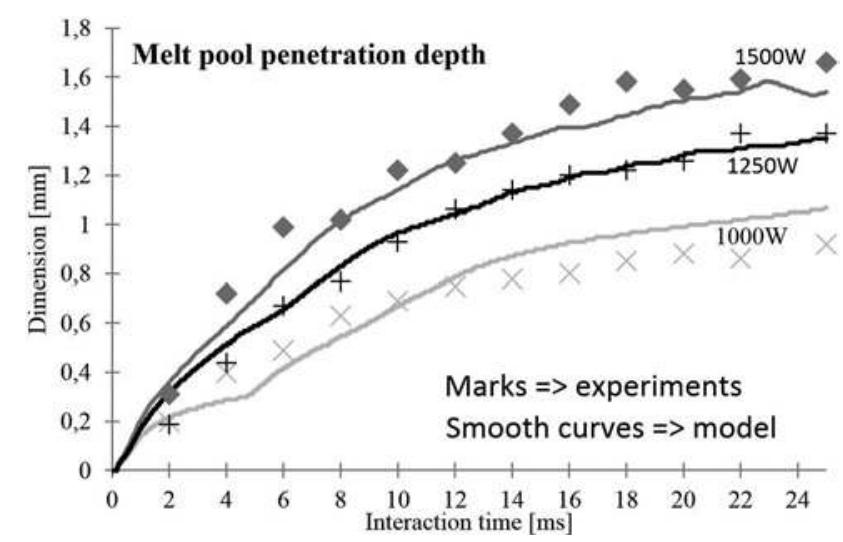

FIG. 7. Time evolution of penetration depth; experiment and numerical results. conditions leading, or not, to the formation of residual porosities. To achieve these goals, a series of static shots were realized by varying the laser power and time of interaction of the laser. The laser used is a disk laser type Nd:YAG $(\lambda=1.06 \mu \mathrm{m})$. The diameter of the focal spot is set to $600 \mu \mathrm{m}$. Shots are operated on DP 600 steel samples with a thickness of $1.8 \mathrm{~mm}$. Cross section macrographs after chemical etching (Béchet-Beaujard) were performed in order to measure the melt pool size evolution.

Figure 6 shows macrographs for a laser power of $1500 \mathrm{~W}$ with an interaction time varying from 2 to $25 \mathrm{~ms}$.

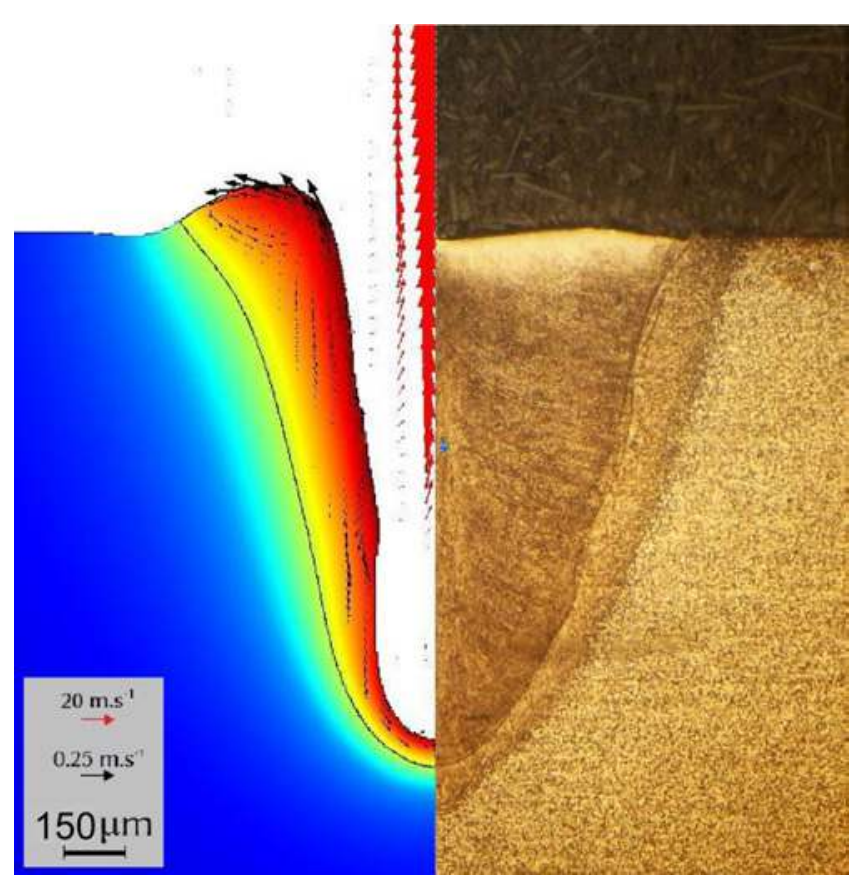

FIG. 8. Comparison of fusion zone shapes given by model and experiment. $P_{\text {laser }}=1250 \mathrm{~W}, t=22 \mathrm{~ms}$. 


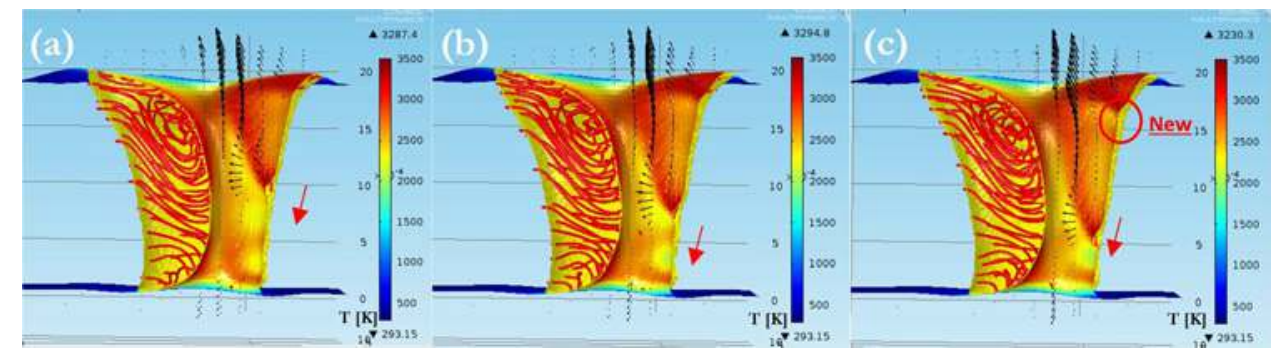

FIG. 9. Shapes of keyhole and melt pool for $4 \mathrm{~kW}$ laser power and $6 \mathrm{~m} / \mathrm{min}$ welding speed (a) $20 \mathrm{~ms}$; (b) $20.1 \mathrm{~ms}$; and (c) $21.2 \mathrm{~ms}$.

The profile of the melted zone is narrow. At high laser powers, residual porosity can be frequently observed. Some porosity can appear at the very beginning of laser irradiation. Figure 6 illustrates, for an interaction time of $6 \mathrm{~ms}$, porosity very close to the base metal. This indicates, because of a high energy density, a very high recoil pressure dominating all other forces. For a power of $1500 \mathrm{~W}$, porosity is present on more than $50 \%$ of shots. This rate drops to less than $10 \%$ for a power of $1000 \mathrm{~W}$. However, the numerical model shows that the keyhole wall becomes vertical when the laser power increases. Therefore, the absorbed laser intensity is low and thus the evaporation process will be reduced. In such condition, the recoil pressure can no longer compensate the capillary pressure, and fluctuations appear at the keyhole wall. This phenomenon favors the formation of porosity during the keyhole collapse process.

Figure 7 shows that the penetration depth for laser powers of 1000,1250 , and $1500 \mathrm{~W}$ increases strongly without stabilization. Note that the numerical model predicts with a good agreement depth penetration with, however, an overestimated width of the melted zone in the early stages. This can be explained by the method used to damp the velocity field in the solid phase. Given the assumptions of the model, the global temporal evolution is satisfactory. Figure 8 shows a comparison of the fusion zone shape given by the model and the experiment. The global shape is well reproduced by the model with similar width at every depth and an equivalent curvature.

\section{THREE-DIMENSIONAL SIMULATION}

Figure 9 shows results obtained with a three-dimensional model for a laser of $4 \mathrm{~kW}$ moving at $6 \mathrm{~m} / \mathrm{min}$. With these parameters and a sample thickness of $1.8 \mathrm{~mm}$, a full penetration welding is observed. Due to the motion of the laser beam, the keyhole geometry becomes asymmetrical with an inclined front, which is then exposed to high intensity laser beam. It can be seen that the model successfully simulates the instabilities on the keyhole front, as observed experimentally by Matsunawa et al..${ }^{17}$ Abt et al., ${ }^{18}$ or Zhang et al. ${ }^{19}$ Figure 9 shows the formation of a hump at the top of the front keyhole wall which propagates along the wall down to the keyhole bottom. When a hump is created at the top of the keyhole, it is exposed to high intensity laser beam. The resulting recoil pressure pushes the hump down along the keyhole wall. While the first hump moves, new humps occur periodically at the keyhole top. The spatial frequency of humps is estimated here at around $200 \mathrm{~Hz}$ and increases with the welding speed. At this point, it is relevant to emphasize that the observed hump evolution is not a mass motion but the phase-front propagation which is determined by the melt ejection toward the sides of the weld pool. It can be observed that an intense jet of vapor is locally generated near the humps. The direction of the ejected vapor plume is then modified by the melt wave. The vapor jet can reach $12 \mathrm{~m} / \mathrm{s}$ when it escapes the keyhole. Note that the multiple reflections are not taken into account for this $3 \mathrm{D}$ simulation. This model requires $24 \mathrm{~h}$ to describe $1 \mathrm{~ms}$ of laser process.

\section{CONCLUSION}

A complete model has been developed to simulate the keyhole formation. This model takes into account melting, evaporation, surface tension, gravity, and calculates temperature, pressure and velocity fields in liquid and gaseous phases. An alternative approach to the existing models is proposed to model laser reflections and energy deposition based on the resolution of Maxwell's equations.

The model gives the deformation of the liquid surface due to the recoil pressure. This pressure is indirectly included in the continuity equation by introducing the evaporation mass flow rate. The interaction between vapor jet and the liquid surface can then be correctly represented without using method such as sharp interface formulation. The results show that the shear stress at the keyhole surface plays a major role on the melt pool dynamics, even in a simple configuration.

The numerical simulations reveal that, under high laser power, bubbles can appear when the laser is shut down during the collapse of the keyhole. Depending on the moment of the laser stop in regard to oscillations, residual porosity may occur. In fact, the initial position of the bubble will determine if the solidification front will have time to capture the bubble. The numerical results have been validated with experimental observations in terms of melt pool dimensions. This model has been extended to a 3D geometry to simulate moving laser beam welding. The humping phenomenon has been successfully simulated. In future work, configurations with different laser powers and welding speeds will be investigated.

${ }^{1}$ J. Lee, S. Ko, D. Farson, and C. Yoo, "Mechanism of keyhole formation and stability in stationary laser welding," J. Phys. D Appl. Phys. 35, 1570-1576 (2002).

${ }^{2} \mathrm{H}$. Ki, P. Mohanty, and J. Mazumder, "Modeling of laser keyhole welding: Part I. Mathematical modeling, numerical methodology, role of recoil pressure; multiple reflections and free surface evolution," Metall. Mater. Trans A 33A, 1817-1830 (2002). 
${ }^{3}$ M. Geiger, H. K. Leitz, H. Koch, and A. Otto, “A 3D transient model of keyhole and melt pool dynamics in laser beam welding applied to the joining of zinc coated sheets," Prod. Eng. Res. Dev. 3, 127-136 (2009).

${ }^{4}$ S. Pang, L. Chen, J. Zhou, Y. Yin, and T. Chen, "A three-dimensional sharp interface model for self-consistent keyhole and weld pool dynamics in deep penetration laser welding," J. Phys. D Appl. Phys. 44, 025301 (2011).

${ }^{5}$ I. W. Cho, J. S. Na, C. Thomy, and F. Vollertsen, "Numerical simulation of molten pool dynamics in high power disk laser welding," J. Mater. Process. Technol. 212, 262-275 (2012).

${ }^{6}$ J. C. Knight, "Theoretical modeling of rapid surface vaporization with back pressure," AIAA J. 17, 519-523 (1979).

${ }^{7}$ R. Fabbro, S. Slimani, F. Coste, and F. Briand, "Analysis of the various melt pool hydrodynamic regimes observed during CW Nd-YAG penetration laser welding," in ICALEO' 2007, Orlando, FL, 2007.

${ }^{8} \mathrm{~W}$. Tan, N. Bailey, and Y. Shin, "Investigation of keyhole plume and molten pool based on a three-dimensional dynamic model with sharp interface formulation," J. Phys. D Appl. Phys. 46, 055501 (2013).

${ }^{9}$ M. Sajid, "A contribution towards the numerical study of bubble dynamics in nucleate boiling at local scale using a conservative level set method," Ph.D. thesis, Université de Cergy-Pontoise, Cergy-Pontoise, 2010.

${ }^{10}$ A. Esmaeeli and G. Tryggvason, "Computations of film boiling. Part I: Numerical method,” Int. J. Heat Mass Trans. 47, 5451-5461 (2004).

${ }^{11} \mathrm{H}$. Ki, S. P. Mohanty, and J. Mazumder, "A numerical method for multiphase incompressible thermal flows with solid-liquid and liquid-vapor phase transformations," Numer. Heat Transfer, Part B 48, 125-145 (2005).
${ }^{12}$ K. Hirano, R. Fabbro, and M. Muller, "Experimental determination of temperature threshold for melt surface deformation during laser interaction on iron at atmospheric pressure," J. Phys. D Appl. Phys. 44, 435402 (2011).

${ }^{13}$ M. Courtois, M. Carin, P. Le Masson, S. Gaied, and M. Balabane, "A new approach to compute multi-reflections of laser beam in a keyhole for heat transfer and fluid flow modeling in laser welding," J. Phys. D Appl. Phys. 46, 505305 (2013)

${ }^{14}$ P. Shcheglov, S. Uspenskiy, A. Gumenyuk, V. Petrovskiy, M. Rethmeier, and V. Yermachenko, "Plume attenuation of laser radiation during high power fiber laser welding," Laser Phys. Lett. 8, 475-480 (2011).

${ }^{15}$ J. Greses, P. Hilton, C. Barlow, and W. Steen, "Plume attenuation under high power Nd:YAG laser welding," in ICALEO' 2002, Scottsdale, AZ, 2002.

${ }^{16}$ T. Schenk, M. I. Richardson, M. Kraska, and S. Ohnimus, "Modeling bulking distortion of DP600 overlap joints due to gas metal arc welding and the influence of the mesh density," Comput. Mater. Sci. 46, 977-986 (2009).

${ }^{17}$ A. Matsunawa, D. J. Kim, N. Seto, M. Mizutani, and S. Katayama, "Dynamics of keyhole and molten pool in laser welding," J. Laser Appl. 10, 247-254 (1998).

${ }^{18}$ F. Abt, M. Boley, R. Weber, T. Graf, G. Popko, and S. Nau, "Novel X-ray system for in-situ diagnostics of laser based processes-First experimental results," Phys. Procedia 12, 761-770 (2011).

${ }^{19}$ M. Zhang, G. Chen, Y. Zhou, and S. Li, "Direct observation of keyhole characteristics in deep penetration laser welding with a $10 \mathrm{~kW}$ fiber laser," Opt. Express 21, 19997-20004 (2013). 